\title{
ULTRA-HIGH PERFORMANCE LIQUID CHROMATOGRAPHIC DETERMINATION OF GENOTOXIC IMPURITIES IN FEBUXOSTAT DRUG SUBSTANCE AND PRODUCTS
}

\author{
BALAJI N*, SAYEEDA SULTANA
}

Department of Chemistry, St. Peter's University, Avadi, Chennai - 600 054, Tamil Nadu, India. Email: priyabalan8380@gmail.com

Received: 26 September 2016, Revised and Accepted: 06 October 2016

\section{ABSTRACT}

Objective: An efficient ultra-high performance liquid chromatographic (UHPLC or Infinity LC 1290) method has been developed and validated for the quantification of possible carcinogenic or genotoxic impurities in febuxostat drug substances and drug products at $18 \mu \mathrm{g} / \mathrm{ml}$ level.

Methods: This method includes the conclusion of four potential genotoxic impurities in febuxostat. The mobile phase is trifluoroacetic acid, acetonitrile, and water with linear gradient elution. The UHPLC column used for the analysis was zorbax RRHD eclipse plus C18 with a length of $100 \mathrm{~mm}$, internal diameter of $2.1 \mathrm{~mm}$, and particle size of $1.8 \mu$.

Results: The limit of detection and limit of quantitation of the impurities are $<0.1(0.00001 \%)$ and $0.3 \mu \mathrm{g} / \mathrm{ml}(0.00003 \%)$ with respect to febuxostat test concentration of $1000 \mu \mathrm{g} / \mathrm{ml}$, respectively. This method has been validated as per ICH guidelines Q2 (R1).

Conclusion: A rapid, cost-effective infinity LC method was wonderfully established for quantitative analysis of possible genotoxic impurities of febuxostat drug substance and drug products.

Keywords: Febuxostat, Genotoxic impurities, Ultra-high performance liquid chromatograph, Infinity-LC 1290, Validation.

(C) 2017 The Authors. Published by Innovare Academic Sciences Pvt Ltd. This is an open access article under the CC BY license (http://creativecommons. org/licenses/by/4. 0/) DOI: http://dx.doi.org/10.22159/ajpcr.2017.v10i1.15381

\section{INTRODUCTION}

Febuxostat is a drug that hinders xanthine oxidase, thus plummeting production of uric acid in the consistency. It is employed in the discussion of chronic gout and hyperuricemia. Febuxostat was revealed by scientists at the Japanese Pharmaceutical Company Teijin in 1998. Teijin united the drug with TAP Pharmaceuticals in the US and Ipsen in Europe. Ipsen obtained a marketing endorsement for febuxostat from the European medicines agency in April 2008, Takeda obtained FDA approval in February 2009, and Teijin attained sanction from the Japanese "Pharmaceuticals and Medical Devices Agency" in 2011. Febuxostat is used to treat chronic gout and hyperuricemia [1]. The National Institute for Health and Clinical Excellence concluded that febuxostat is extra effective than usual dosages of allopurinol, but not more effective than higher doses of allopurinol [1]. Febuxostat is in the US pregnancy category $\mathrm{C}$; there are no adequate and well-controlled studies in pregnant women [2].

The determination of febuxostat and its related compounds were performed by LC studies at the level of $1000 \mu \mathrm{g} / \mathrm{ml}$ or $0.10 \% \mathrm{w} / \mathrm{w}$ with respect to the target analyte concentration. As per the selection of synthetic route, the related compounds of febuxostat may be categorized as genotoxic or carcinogenic. Nowadays, the regulators were very much interested about the genotoxic impurities present in drug substances and drug products. They assert to have a control of potential genotoxic impurities in the synthetic drug process. The control of potential genotoxic impurities shall be provided based on the availability of the high sensitive chromatography method. This directs us to evolve a method for the genotoxic impurities of febuxostat in the drug substances and drug products using ultra-high performance liquid chromatographic (UHPLC)

Other subjects were explained about the determination of febuxostat was performed by liquid chromatograph coupled mass spectrometer (LCMS/MS) in human plasma [3-6], by LC-ultraviolet (UV) [7], by HPLC [8-10], by UPLC [11-13], and by UPLC/MS in dog plasma [14]. The related compounds of febuxostat were separated and determined at the level $0.10 \% \mathrm{w} / \mathrm{w}$ level or $1000 \mu \mathrm{g} / \mathrm{ml}$ level by UPLC in literature [13] Hence, as to increase the sensitivity of the method using these four genotoxic impurities in febuxostat, the research work has been initiated which was grounded in the literature [13,15-24,26-29].

As per the literature survey of the febuxostat drug substances and drug products, no one has reported the infinity LC/UHPLC method for the quantification of probable genotoxic impurities in febuxostat at $18 \mu \mathrm{g} / \mathrm{ml}$ level, and this is the novelty of the article.

\section{Evaluation study of genotoxicity and carcinogenicity}

From the appraisal study of genotoxic and carcinogenic impurities imp-1, imp-2, imp-3, and imp-4 (Fig. 1) have been given an alert for potential carcinogen which was due to the presence of aldehyde functional group in its own structure of the molecule and also based on quantitative structure-activity relationship (QSAR). These alerts have been obtained from the Toxtree software and its version was 2.6.6. The recommended maximum daily dosage of febuxostat is about $80 \mathrm{mg}$. The threshold of toxicological concern limit could be $18.75 \mu \mathrm{g} / \mathrm{ml}$ as per the calculation provided in ICH guideline M7 and based on acceptable intake of $1.5 \mu \mathrm{g}$ /day was considered to be protective for a lifetime of daily exposure.

\section{METHODS}

\section{Instrumentation}

An Agilent - 1290 series UHPLC/infinity LC consisting of a binary/quaternary pump, column compartment (Zorbax RRHD eclipse plus C18 with $100 \mathrm{~mm} \times 2.1 \mathrm{~mm}, 1.8 \mu \mathrm{m}$ was used as a column), autoinjector, and a diode array detector (M/s. Agilent Technologies, USA). Water bath equipped with controller (Amkette Analytics, ANM alliance) was used for forced degradation studies. Photolytic studies were carried out in a photostability chamber (Thermolab Photo Stability Chamber, India). Thermal degradation works were accomplished in a hot air oven (Amkette Analytics, ANM alliance). 


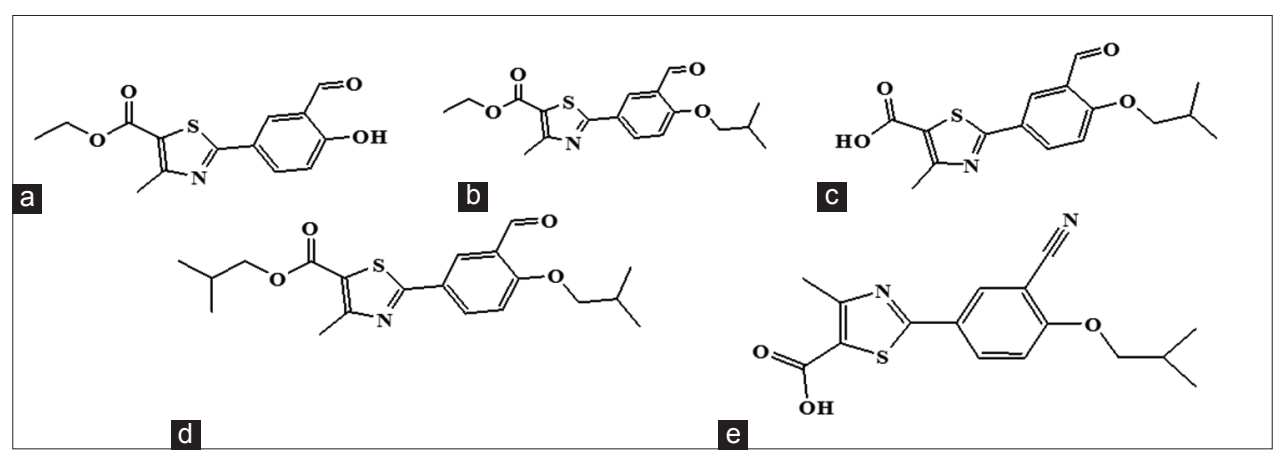

Fig. 1: The structure of potential genotoxic imurities and febuxostat. (a) ethyl 2-(3-formyl-4-hydroxyphenyl)-4-methylthiazole5-carboxylate or imp-1, (b) ethyl 2-(3-formyl-4-isobutoxyphenyl)-4-methylthiazole-5-carboxylate or imp-2, (c) 2-(3-formyl-4isobutoxyphenyl)-4-methylthiazole-5-carboxylic acid or imp-3, (d) isobutyl 2-(3-formyl-4-isobutoxyphenyl)-4-methylthiazole-5carboxylate or im-4, (e) 2-(3-cyano-4-isobutoxy-phenyl)-4-methyl-thiazole-5-carboxylic acid or febuxostat

\section{Reagents and chemicals}

Febuxostat, imp-1, imp-2, imp-3, and imp-4 (Fig. 1) were synthesized in chemistry lab, St. Peter's university. Trifluoroacetic acid and acetonitrile were bought from Fisher Scientific. HPLC grade water was used, equipped with the Elga water purification system, Metrohm.

\section{Solutions preparation Sample diluent}

The sample diluent was prepared by mixing $300 \mathrm{ml}$ of mobile Phase A solution and $700 \mathrm{ml}$ of mobile Phase B solution. Filtered and degassed for usage of analysis.

\section{Standard solution}

The standard solution was prepared by exactly weighed and transferred about $18 \mathrm{mg}$ each of imp-1,imp-2, imp-3, imp-4 and febuxostat in $100 \mathrm{ml}$ volumetric flask; added $50 \mathrm{ml}$ of sample diluent and ultra-sonicated for 5 minutes. After sonication, the solution was made up to the mark with the sample diluent. Further, $10 \mathrm{ml}$ of this solution was diluted into a $100 \mathrm{ml}$ volumetric flask and made up to the mark with sample diluent. Hence, the standard solution concentration was $18 \mu \mathrm{g} / \mathrm{ml}$ with respect to the test concentration of $1000 \mu \mathrm{g} / \mathrm{ml}$ febuxostat. The solution was centrifuged at $25^{\circ} \mathrm{C}$ for 30 minutes and filtered through $0.22 \mu \mathrm{m}$ syringe filter and injected. By keeping, the same solution at ambient temperature for $24 \mathrm{hrs}$ and it was used for solution stability study.

\section{Sample solution}

The sample solution was prepared by accurately weighed and transferred about $10000 \mathrm{mg}$ of sample into $10 \mathrm{ml}$ volumetric flask; added $5 \mathrm{ml}$ of sample diluent and ultra-sonicated for 5 minutes. After sonication, the solution was made up to the mark with the sample diluent. The solution was centrifuged at $25^{\circ} \mathrm{C}$ for 30 minutes and filtered through $0.22 \mu \mathrm{m}$ syringe filter and injected. By keeping, the same solution at ambient temperature for $24 \mathrm{hrs}$ and it was used for solution stability study.

\section{Method development}

Several methods have been developed by HPLC and UPLC for the determination of febuxostat in the bulk and formulated products. Some developed methods for the quantification of febuxostat and its related compounds in the bulk and formulated products by UPLC $[3,8-14,26]$. These previously published research articles were failed to explain about the probable genotoxic impurities in febuxostat. Actually, the four impurities of febuxostat, which were mentioned in Fig. 1, were evaluated as potential genotoxic impurities using QSAR guidelines and these impurities were determined at $1500 \mu \mathrm{g} / \mathrm{ml}$ level in febuxostat by UPLC as per the literature work.

This mainly leads us to do further development of the method with four genotoxic impurities in febuxostat at the $18 \mu \mathrm{g} / \mathrm{ml}$ level by UHPLC. The same method [13] has been used for the initial development, but the sensitivity of the method was found at the very lowest level of those impurities of the literature work [13]. Hence, as to increase the sensitivity of the method, the mobile phase, $\mathrm{pH}$ and gradient composition have been modified accordingly. The $\mathrm{pH}$ of the mobile phase was maintained in the acidic region based on the literature [13], which was achieved by the addition of $1 \mathrm{ml}$ of trifluoroacetic acid in $1000 \mathrm{ml}$ of water. The mass compatible mobile phase has been selected for the development and validation, because if any impurities were detected at the level of above or below limit of quantification (LOQ) in the sample which needs to be confirmed using UPLC-MS study.

Fortunately, these four potential genotoxic impurities were not present/not detected in the samples of drug substances and drug products. Hence, these samples were not screened by UPLC-MS. This could be the limitation of the present research work. These impurities have been eluted and separated well within the time of 6 minutes, but to ensure the consistency and specificity of the other impurities which was mentioned in the research article [26]; the gradient program has been slightly modified and extended up to 12 minutes. From this trial, the method was specific for all the impurities which have mentioned in the research article [26]. Furthermore, impurities of V and VI [26] were matching and other impurities were not matching with the present research work; hence, totally these four impurities considered for the research work. The results and comparisons of previously published articles were given in Table 1.

\section{Concluded method for validation purpose}

The UHPLC column was used Zorbax RRHD C18, $100 \mathrm{~mm} \times 2.1 \mathrm{~mm} \times$ $1.8 \mu \mathrm{m}$. The mobile Phase A was diluted $1 \mathrm{ml}$ of trifluoroacetic acid in $1000 \mathrm{ml}$ of water. The mobile Phase B was acetonitrile. The gradient program was mentioned as $\mathrm{min} / \% \mathrm{~B}$ composition; 0.00/5.0, 0.80/5.0, $2.00 / 25.0,2.40 / 35.0,6.00 / 50.0,6.40 / 65.0,8.00 / 80.0,9.00 / 90.0$, $10.00 / 90.0,10.20 / 5.0$, and $12.00 / 5.0$. The wavelength of detection was $320 \mathrm{~nm}$ and injection volume was $2 \mu$ l. The column compartment temperature was maintained at $45^{\circ} \mathrm{C}$.

\section{Method validation}

Once chromatographic conditions were established; the method was validated in compliance with ICH guidelines. The following parameters of system suitability, specificity, linearity, precision, accuracy, limit of detection (LOD), LOQ, robustness, and forced degradation studies were performed for validation.

\section{System suitability}

The standard solution was prepared using standard as per the method. Moreover, six replicates are injected into the system.

\section{Specificity}

The specificity of the method was determined by comparing chromatograms obtained from standard, blank and that of forced degradation studies. 
Table 1: Comparative of results with the previously published research works

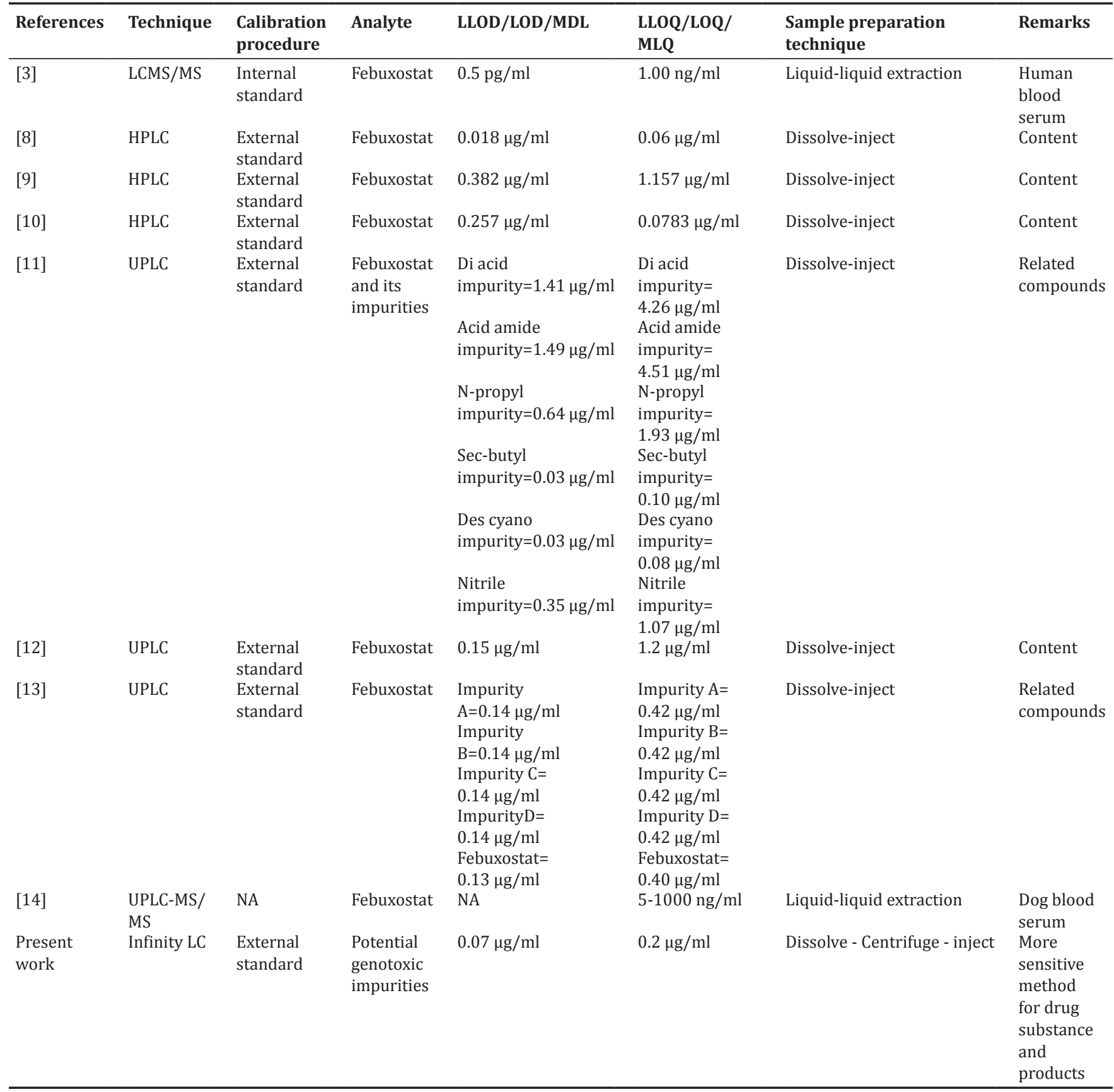

LCMS/MS: Liquid chromatograph coupled mass spectrometer, HPLC: High performance liquid chromatography, UPLC: Ultra performance liquid chromatograph, UPLCMS/MS: Ultra performance liquid chromatography coupled mass spectrometer

\section{Linearity}

The linearity of detector response was established by plotting a graph between concentrations versus average area responses of the analytes.

\section{Precision}

Six replicates injections of the standard solution were injected into the UHPLC system as considered as system precision. The precision of test method was evaluated by six preparations of spiked sample solutions with impurities of imp-1, imp-2, imp-3, and imp-4 from the same batch as considered as method precision.

\section{Accuracy}

A study of accuracy (recovery) was performed by spiking genotoxic impurities in drug substances and drug products. Samples were prepared as per the proposed method at QL-150\% of the target test concentration.

\section{LOD and LOQ limits}

The LOD and LOQ were examined based on signal-to-noise ratio method as per the ICH guideline Q2 (R1). The signal to noise ratio for LOD is 3:1 and the LOQ is 10:1. This was performed by performing the sequence of dilute solutions with a known concentration LOD and LOQ has been determined.

\section{Robustness}

Robustness of the method was investigated by varying the instrumental conditions such as flow rate $( \pm 0.1 \mathrm{ml} / \mathrm{min})$, column oven temperature $\left( \pm 5^{\circ} \mathrm{C}\right)$, and the addition of trifluoroacetic acid in mobile phase 
$( \pm 0.2 \mathrm{ml})$. The standard solution was prepared and analyzed as per the test procedure monitored the system suitability results.

\section{Forced degradation studies \\ Acid degradation studies}

Accurately weighed and transferred about 50,000 mg of sample into a $50 \mathrm{ml}$ of volumetric flask. Added $30 \mathrm{ml}$ of diluent and $10 \mathrm{ml}$ of $0.4 \mathrm{~N}$ $\mathrm{HCl}$ was added and refluxed at $60^{\circ} \mathrm{C}$ for $24 \mathrm{~h}$ and neutralized with 0.4 $\mathrm{N} \mathrm{NaOH}$. After the exposure, the resultant solution was made up to the mark with diluent. The solution was centrifuged at $25^{\circ} \mathrm{C}$ for $30 \mathrm{~min}$ and filtered through $0.22 \mu \mathrm{m}$ syringe filter and injected into the system, and chromatograms were recorded to assess the stability of the sample.

\section{Alkali degradation studies}

Accurately weighed and transferred about 50,000 mg of sample into a $50 \mathrm{ml}$ of volumetric flask. Added $30 \mathrm{ml}$ of diluent and $10 \mathrm{ml}$ of $0.4 \mathrm{~N}$ $\mathrm{NaOH}$ was added and refluxed at $60^{\circ} \mathrm{C}$ for $24 \mathrm{hrs}$ and neutralized with $0.4 \mathrm{~N} \mathrm{HCl}$. After the exposure, the resultant solution was made up to the mark with diluent. The solution was centrifuged at $25^{\circ} \mathrm{C}$ for 30 minutes and filtered through $0.22 \mu \mathrm{m}$ syringe filter and injected into the system, and chromatograms were recorded to assess the stability of the sample.

\section{Oxidative degradation}

Accurately weighed and transferred about 50,000 mg of sample into a $50 \mathrm{ml}$ of volumetric flask. Added $30 \mathrm{ml}$ of diluent and $10 \mathrm{ml}$ of $3 \% \mathrm{H}_{2} \mathrm{O}_{2}$ was added and kept at room temperature for $24 \mathrm{hrs}$. After the exposure, the resultant solution was made up to the mark with diluent. The solution was centrifuged at $25^{\circ} \mathrm{C}$ for 30 minutes and filtered through $0.22 \mu \mathrm{m}$ syringe filter and injected into the system, and chromatograms were recorded to assess the stability of the sample.

\section{Dry heat degradation}

Accurately weighed and spread $10 \mathrm{~g}$ of sample in a dried Petri dish uniformly (maximum of $5 \mathrm{~mm}$ thickness). Placed the dish in an oven at $60^{\circ} \mathrm{C}$ for 7 days without vacuum. Recorded the weight (petri dish + sample) and noted the appearance before and after the stress test. Calculated percent change for the weight of sample. Weighed and transferred about 50,000 mg of stressed sample into a $50 \mathrm{ml}$ of volumetric flask. After the exposure, the resultant solution was made up to the mark with diluent. The solution was centrifuged at $25^{\circ} \mathrm{C}$ for 30 minutes and filtered through $0.22 \mu \mathrm{m}$ syringe filter and injected into the system, and chromatograms were recorded to assess the stability of the sample.

\section{Photostability studies}

Weighed and transferred about 50,000 mg of exposed sample into two $50 \mathrm{ml}$ of quartz volumetric flasks and the solution was made up to the mark with diluent. One quartz volumetric flask was fully covered with aluminum foil and labeled as control sample solution, and another quartz volumetric flask was kept without covering aluminum foil and marked as stressed sample solution. Placed the tightly sealed flask in a suitable chamber and irradiate first under fluorescent light for a total exposure of $1.2 \times 10^{6}$ Lux hours, and then under UV fluorescent light for a total exposure of $200 \mathrm{~W} . \mathrm{hr} / \mathrm{m}^{2}$. The solutions were centrifuged at $25^{\circ} \mathrm{C}$ for 30 minutes and filtered through $0.22 \mu \mathrm{m}$ syringe filter and injected into the system, and chromatograms were recorded to assess the stability of the sample.

\section{RESULTS AND DISCUSSION}

Different chromatographic conditions were employed for the analysis of febuxostat in both bulk and pharmaceutical dosage forms. The comparative results of previously published articles were given in Table 1.

The standard solution was injected repeatedly and performed the calculation of \% relative standard deviation (RSD) for each peak and values were obtained was $<5 \%$. This indicates that the equipment was accurate and fit for study. The system precision results have been given in Table 2. The Chromatographs of blank and standard have been shown in Fig. 2.

The \%RSD for the content of imp-1, imp-2, imp-3, and imp-4 was obtained below $2 \%$. The $\%$ RSD for content impurities was within $3 \%$ for intermediate precision which was performed by different analysts, column, instrument, and day. This result shows that the method was precise. Table 3 shows the results of method precision data.

The LOD for imp-1, imp-2, imp-3, imp-4 and febuxostat were 0.070 , $0.076,0.080,0.070$ and $0.073 \mu \mathrm{g} / \mathrm{ml}$, respectively. The LOQ for imp-1,

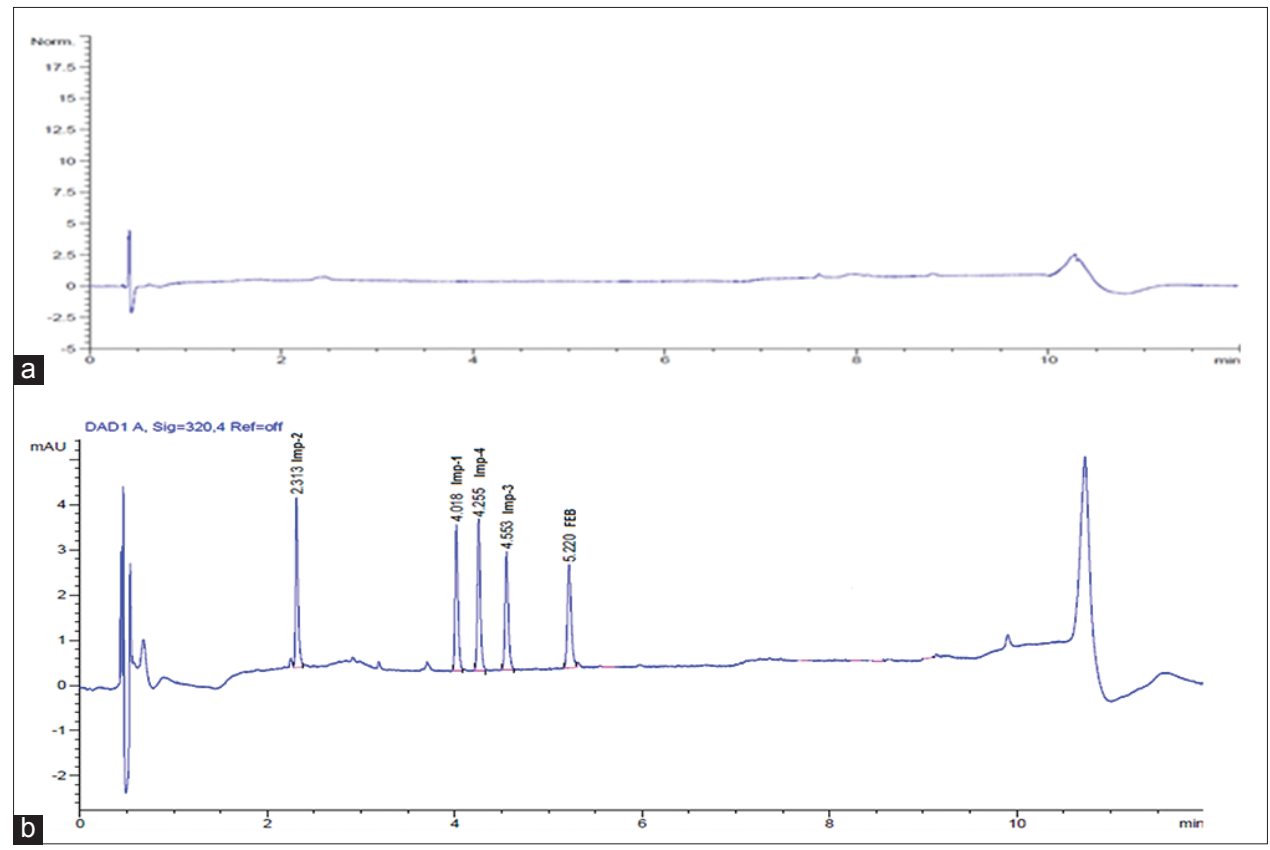

Fig. 2: ( $a$ and b) Chromatograms of blank and standard solution 
imp-2, imp-3, imp-4 and febuxostat were $0.21,0.23,0.24,0.21$ and $0.22 \mu \mathrm{g} / \mathrm{ml}$, respectively. The LOD and LOQ results indicated that the method was most sensitive when compared to the reported methods shown in Table 1.

The correlation coefficient values have been shown in Tables 4-8. The linearity graphs were shown in Figs. 3-7. The values of the correlation coefficients were almost equal to one; this indicates that the developed method was linear. The regression results indicate that the validated method was linear over the total concentration and it was satisfactory for its concentration range from LOQ to $150 \%$. The percentage of bias was calculated by multiplying the slope with 100 and then the resultant was divided by the reference value, which can be considered as the area response obtained at the $100 \%$ level. The calculated $\%$ of bias values indicates that the method was linear and it was very close to the origin or close to the ideal theoretical value.

The accuracy percentage for imp-1, imp-2, imp- 3 and imp- 4 were $85-105 \%$ of drug substance and drug products. This result indicates that the method was accurate and appropriate as the mean accuracy value was within the limit $(80-120 \%)$.

The specificity of the method was demonstrated by the peak purity (i.e., the purity angle is lesser than the purity threshold) using the diode array detector for degraded samples. The peak purity analysis was homogeneous for imp-1, imp-2, imp-3, and imp-4. There was no interference was observed from blank peaks and impurities. There was no secondary peak aroused from forced degraded samples. The results of forced degradation study indicate that the method was stability indicating and the impurities, viz., imp-1, imp-2, imp-3 and imp-4 were not the degradation impurities, and these are process related impurities only.

By carefully varying in chromatographic conditions the resolution between febuxostat, imp-1, imp-2, imp-3 and imp-4 were evaluated. The resolution between impurities and febuxostat was obtained $>1.8$

Table 2: System precision results of imp-1, imp-2, imp-3, imp-4 and febuxostat

\begin{tabular}{llllll}
\hline \multirow{2}{*}{ Injection } & \multicolumn{2}{l}{ Area observed } \\
\cline { 2 - 6 } & Imp-1 & Imp-2 & Imp-3 & Imp-4 & Febuxostat \\
\hline 1 & 145,204 & 109,528 & 92,163 & 97,921 & 65,121 \\
2 & 144,956 & 110,785 & 93,001 & 97,810 & 68,265 \\
3 & 145,060 & 111,527 & 92,964 & 97,777 & 69,842 \\
4 & 145,329 & 112,328 & 92,763 & 97,707 & 70,798 \\
5 & 145,239 & 111,833 & 93,521 & 97,917 & 71,827 \\
6 & 145,110 & 112,045 & 92,661 & 97,811 & 72,287 \\
Mean & 145,150 & 111,341 & 92,846 & 97,824 & 69,690 \\
SD & 134.32 & 1033.69 & 447.48 & 82.85 & 2663.39 \\
\%RSD & 0.09 & 0.93 & 0.48 & 0.08 & 3.82 \\
Limit & RSD should be<15\% & & & \\
\hline
\end{tabular}

Imp: Impurity, SD: Standard deviation, RSD: Relative standard deviation in all the varied chromatographic conditions carried out (flow rate, the addition of trifluoroacetic acid and column temperature). The result of robustness analysis shows that the method was considered robust.

\section{ACKNOWLEDGMENTS}

The authors acknowledge the support provided by the research scholars of the chemistry department, St. Peter's University.

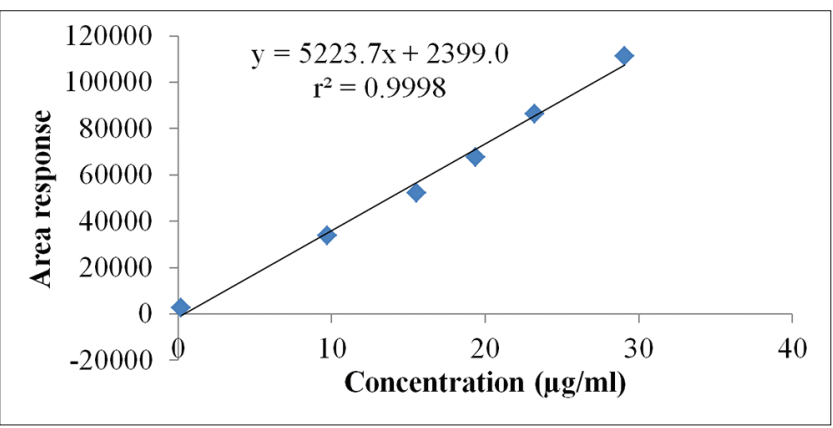

Fig. 3: Linearity graph for imp-1

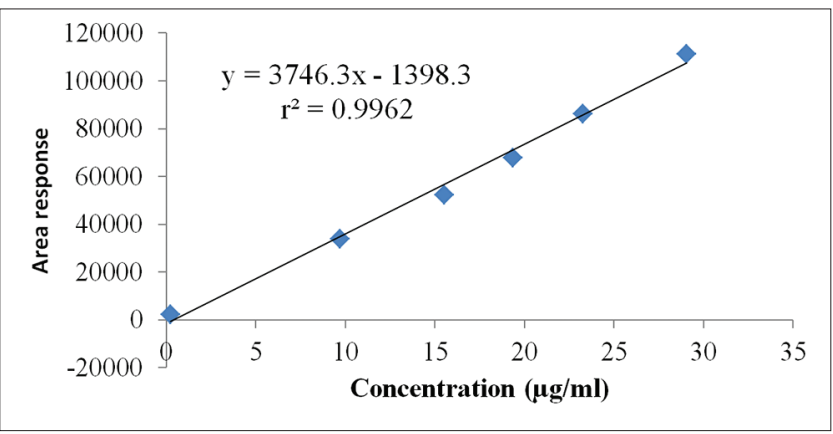

Fig. 4: Linearity graph for imp-2

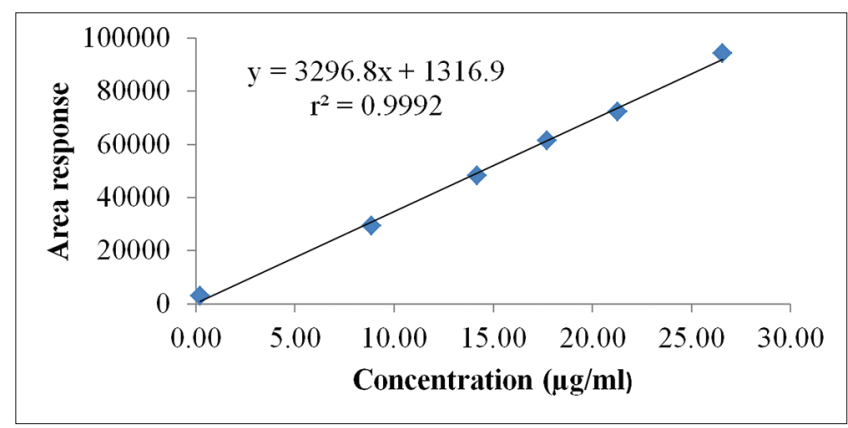

Fig. 5: Linearity graph for imp-3

Table 3: Method precision results of imp-1, imp-2, imp-3 and imp-4

\begin{tabular}{llll}
\hline Preparation & Imp- $\mathbf{~ c o n t e n t ~}(\boldsymbol{\mu g} / \mathbf{m l})$ & Imp-2 content $(\boldsymbol{\mu g} / \mathbf{m l})$ & Imp-3 content $(\boldsymbol{\mu g} / \mathbf{m l})$ \\
\hline 1 & 17.552 & 18.025 & 17.956 \\
2 & 17.668 & 18.158 & 17.865 \\
3 & 17.023 & 18.026 & 17.952 \\
4 & 17.258 & 18.251 & 17.874 \\
5 & 17.325 & 18.036 & 18.025 \\
6 & 17.125 & 18.325 & 18.001 \\
Mean & 17.3 & 18.1 & 17.9 \\
SD & 0.25 & 0.13 & 0.07 \\
\%RSD & 1.4 & 0.7 & 0.4 \\
Limit & RSD for content should be<5\% & & 17.996 \\
\hline
\end{tabular}

Imp: Impurity, SD: Standard deviation, RSD: Relative standard deviation 


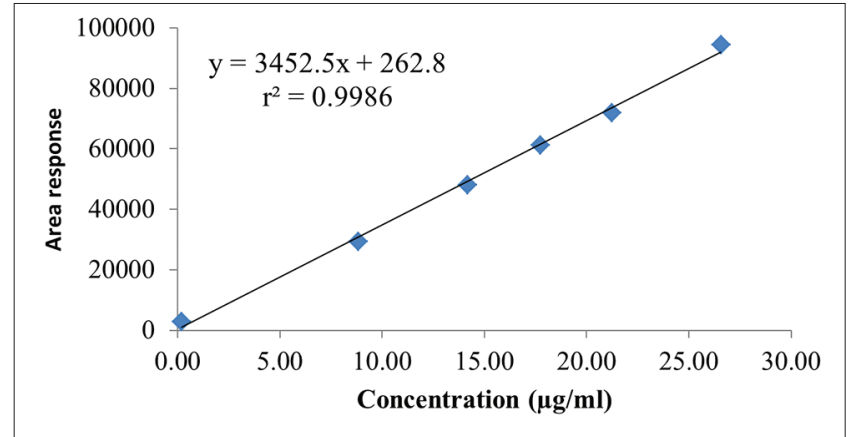

Fig. 6: Linearity graph for imp-4

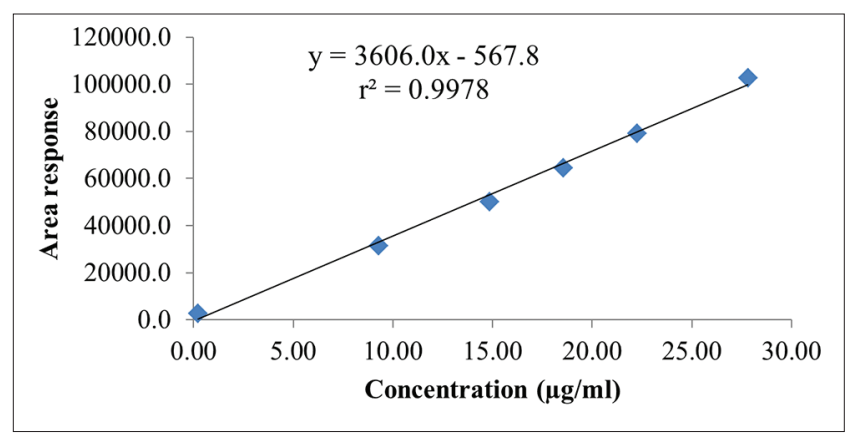

Fig. 7: Linearity graph for febuxostat

Table 4: Linearity data of imp-1

\begin{tabular}{llll}
\hline Sample & \% Level & Concentration $(\boldsymbol{\mu g} / \mathbf{m l})$ & Peak response \\
\hline 1 & LOQ & 0.21 & 4385 \\
2 & 50 & 9.0 & 48,745 \\
3 & 80 & 14.5 & 77,380 \\
4 & 100 & 18.1 & 96,529 \\
5 & 120 & 21.6 & 115,521 \\
6 & 150 & 27.2 & 145,150 \\
Slope & 5223.7 & \\
Y-intercept & 2399.0 & \\
Correlation & 0.9998 & \\
co-efficient & & \\
square (r $\left.{ }^{2}\right)$ & & \\
Bias (\%) & 5.4 & \\
\hline
\end{tabular}

LOQ: Limit of quantification and each \% level injected twice

Table 5: Linearity data of imp-2

\begin{tabular}{llll}
\hline Sample & \% Level & Concentration $(\boldsymbol{\mu g} / \mathbf{m l})$ & Peak response \\
\hline 1 & LOQ & 0.23 & 2800 \\
2 & 50 & 9.68 & 34,200 \\
3 & 80 & 15.50 & 52,396 \\
4 & 100 & 19.36 & 68,000 \\
5 & 120 & 23.24 & 86,522 \\
6 & 150 & 29.06 & 111,341 \\
Slope & & 3746.3 & \\
Y-intercept & -1398.3 & \\
Correlation & 0.9962 & \\
coefficient & & \\
square ( $\left.{ }^{2}\right)$ & & \\
Bias (\%) & 5.5 & \\
\hline
\end{tabular}

LOQ: Limit of quantification and each level injected twice

\section{CONCLUSION}

A swift, economical infinity LC method was magnificently established for quantitative analysis of possible genotoxic impurities of febuxostat
Table 6: Linearity data of imp-3

\begin{tabular}{llll}
\hline Sample & \% Level & Concentration $(\mu \mathrm{g} / \mathrm{ml})$ & Peak response \\
\hline 1 & $\mathrm{QL}$ & 0.24 & 3727 \\
2 & 50 & 9.108 & 29,639 \\
3 & 80 & 14.562 & 48,979 \\
4 & 100 & 18.216 & 60,597 \\
5 & 120 & 21.852 & 73,121 \\
6 & 150 & 27.324 & 92,845 \\
Slope & 3296.8 & \\
Y-intercept & 1316.9 & \\
Correlation & & 0.9992 & \\
coefficient & & \\
square (r $\left.{ }^{2}\right)$ & & \\
Bias (\%) & 5.4 & \\
\hline
\end{tabular}

LOQ: Limit of quantification and each level injected twice

Table 7: Linearity data of imp-4

\begin{tabular}{llll}
\hline Sample & \% Level & Concentration $(\boldsymbol{\mu g} / \mathbf{m l})$ & Peak response \\
\hline 1 & QL & 0.21 & 2890.5 \\
2 & 50 & 8.86 & $29,183.6$ \\
3 & 80 & 14.16 & $48,165.6$ \\
4 & 100 & 17.71 & $61,301.2$ \\
5 & 120 & 21.25 & $72,133.0$ \\
6 & 150 & 26.57 & $94,285.9$ \\
Slope & 3452.5 & \\
Y-intercept & 262.8 & \\
Correlation & 0.9986 & \\
coefficient & & \\
square (r $\left.{ }^{2}\right)$ & & & \\
Bias (\%) & 5.6 & \\
\hline
\end{tabular}

LOQ: Limit of quantification and each level injected twice

Table 8: Linearity data of febuxostat

\begin{tabular}{llll}
\hline Sample & \% Level & Concentration $(\boldsymbol{\mu g} / \mathbf{m l})$ & Peak response \\
\hline 1 & QL & 0.22 & 2845.3 \\
2 & 50 & 9.27 & $31,691.8$ \\
3 & 80 & 14.83 & $50,280.8$ \\
4 & 100 & 18.54 & $64,650.6$ \\
5 & 120 & 22.24 & $79,327.5$ \\
6 & 150 & 27.81 & $102,813.5$ \\
Slope & 3606.0 & \\
Y-intercept & -567.8 & \\
Correlation & 0.9978 & \\
coefficient & & \\
square (r $\left.{ }^{2}\right)$ & & & \\
Bias $(\%)$ & & 5.6 & \\
\hline
\end{tabular}

LOQ: Limit of quantification and each level injected twice

drug substance and drug products. The method was found to be specific and truthful with attired and constant recoveries. The valid method may be used for the regular analysis of the determination of potential genotoxic impurities of febuxostat drug substances and drug products in quality control laboratories.

\section{REFERENCES}

1. Febuxostat for the Management of Hyperuricemia in People with Gout (TA164). Ch. 4. Consideration of the Evidence. Available from: https:// www.nice.org.uk/guidance/ta164/chapter/4-consideration-of-theevidence.

2. ULORIC (Febuxostat) Tablet for Oral Use Initial U.S. Approval; 2009. Available from: http://www.accessdata.fda.gov/drugsatfda_docs/ label/2009/021856lbl.pdf.

3. Chandu BR, Kanala K, Hwisa NT, Katakam P, Khagga M. Bioequivalance and pharmacokinetic study of febuxostat in human plasma by using LC-MS/MS with liquid liquid extraction method. 
Springerplus 2013;2(1):194.

4. Shi Z, Liu J, Hu XJ, Tu JZ. Development of a simple LC-MS/MS method for the determination of febuxostat in human plasma and its application to a bioequivalence study. Pharmazie 2013;68(6):396-400.

5. Vaka VR, Inamadugu JK, Pilli NR, Ramesh M, Katreddi HR. A sensitive LC-MS/MS method for the quantification of febuxostat in human plasma and its pharmacokinetic application. Biomed Chromatogr 2013;27(11):1406-12.

6. Lukram O, Parmar S, Hande A. Determination of febuxostat in human plasma using ultra-performance liquid chromatography tandem mass spectrometry. Drug Test Anal 2013;5(6):492-9.

7. Younes KM, El-Kady EF, Elzanfaly ES. Determination of febuxosta in human plasma using RP-LC-UV method. J Chromatogr Sci 2016;54(6):1022-7.

8. Rao KN, Ganapaty S, Rao LA. Development and validation of RPHPLC method for estimation of febuxostat in bulk and tablet dosage form. Int J Res Pharm Chem 2012;2(4):1104-8.

9. Ravisankar P, Rani AK, RamanaVK, Gowthami S. Developmen and validation of rapid RP - HPLC method for the determination of febuxostat (a non-purine selective xanthine-oxidase/xanthinedehydrogenase inhibitor) in bulk and pharmaceutical dosage form. World J Pharm Pharm Sci 2015;4(9):1046-61.

10. Mukthinuthalapati MA, Bandaru SP, Bukkapatnam V, Mohapatro C Development and validation of a stability-indicating RP-HPLC method for the determination of febuxostat (a xanthine oxidase inhibitor) J Chromatogr Sci 2013;51(10):931-8.

11. Molleti S, Rao V, Jayaveera KN. A stability indicating RP-UPLC method for estimation of febuxostat and its impurities in bulk drugs and pharmaceutical dosage forms. Am J Pharm Tech Res 2013;3(2):405-22.

12. Sahu K, Shaharyar M, Siddiqui SA. Establishment of the inheren stability of febuxostat and development of a validated stabilityindicating method by UPLC according to ICH requirement. Med Chem Res 2013;22(4):1641-7.

13. Musirike MR, Reddy HK, Mallu UR. Development and validation of reverse phase-ultra performance liquid chromatographic method for estimation of related substances in febuxostat drug substance. Pharm Anal Acta 2015;6(10):1-6.

14. Zhang T, Sun Y, Zhang P, Gao J, Wang S, He Z. Ultra-performance liquid chromatography-tandem mass spectrometry method for the determination of febuxostat in dog plasma and its application to a pharmacokinetic study. Biomed Chromatogr 2013;27(2):137-41.

15. Pharmaceutical Development Q8(R2). Available from: http://www. ich.org/fileadmin/Public_Web_Site/ICH_Products/Guidelines/Quality/
Q8 R1/Step4/Q8 R2 Guideline.pdf.

16. Vogt FG, Kord AS. Development of quality-by-design analytical methods. J Pharm Sci 2011;100(3):797-812.

17. Reid GL, Morgado J, Barnett K, Harrington B, Wang J, Harwood J, et al Analytical quality by design (AQbD) in pharmaceutical development. Am Pharm 2013;16:49-59.

18. Debrus B, Guillarme D, Rudaz D. Improved quality-by-design compliant methodology for method development in reverse-phase liquid chromatography. J Pharm Biomed Anal 2013;84:215-23.

19. Validation of Analytical Procedures: Text and Methodology Q2(R1) Available from: http://www.ich.org/fileadmin/Public_Web_Site/ICH Products/Guidelines/Quality/Q2 R1/Step4/Q2 R1 Guideline.pdf.

20. Preventing Occupational Exposure to Antineoplastic and Other Hazardous Drugs in Health Care Settings Safety and Health, DHHS (NIOSH). Available from: https://www.cdc.gov/niosh/docs/2004-165/.

21. OECD Quantitative Structure-Activity Relationships Project [(Q) SARs]. Available from: http://www.oecd.org/chemicalsafety/testing/ oecdquantitativestructure-activityrelationshipsprojectqsars.htm.

22. Roy K, Kar S, Das RN. Understanding the Basics of QSAR for Applications in Pharmaceutical Sciences and Risk Assessment. New York: Academic Press, Elsevier; 2015.

23. Stability Testing: Photostability Testing of New Drug Substances and Products Q1B. Available from: http://www.ich.org/fileadmin/ Public_Web_Site/ICH_Products/Guidelines/Quality/Q1B/Step4/Q1B_

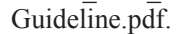

24. Assessment and Control of DNA Reactive (Mutagenic) Impurities in Pharmaceuticals to Limit Potential Carcinogenic Risk M7. Available from: http://www.ich.org/fileadmin/Public Web Site/ICH Products/ Guidelines/Multidisciplinary/M7/M7_Step_4.pdf.

25. Available from: http://www.drugbank.ca/drugs/DB04854.

26. Ravi P, Shanmugam V, Lahoti AM, Deepthi PS, Innareddy V, Rao S, et al. Investigation of various impurities in febuxostat. Pharm Technol 2014;38(9):1-6.

27. Balaji N, Sayeeda S. Trace level determination and quantification of potential genotoxic impurities in dasatinib drug substance by UHPLC/ infinity LC. Int J Pharm Pharm Sci 2016;8(10):209-16.

28. Mercy R, Suja R, Caroling G, Tiwari S. In vitro evaluation of antioxidant, antimicrobial, anticancer activities and characterization of Brassica oleracea. Var. Bortrytis. L synthesized silver nanoparticles. Int J Pharm Pharm Sci 2013;5(4):239-51.

29. Manisha B, Priyanka P. Development and evaluation of paclitaxel loaded nanoparticles using 24 factorial design. Int J Curr Pharm Res 2015;7(2):64-72. 